\title{
TBD: Trajectory-Based Data Forwarding for Light-Traffic Vehicular Networks
}

\author{
Jaehoon Jeong, Shuo Guo, Yu Gu, Tian He and David Du \\ Department of Computer Science \& Engineering, University of Minnesota \\ Email: \{jjeong,sguo,yugu,tianhe,du\}@cs.umn.edu
}

\begin{abstract}

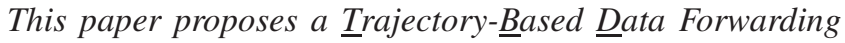
$(T B D)$ scheme, tailored for the data forwarding in lighttraffic vehicular ad-hoc networks. State-of-the-art schemes have demonstrated the effectiveness of their data forwarding strategies by exploiting known vehicular traffic statistics (e.g., densities and speeds) in these vehicular networks. These results are encouraging, however, further improvements can be made by taking advantage of the growing popularity of GPSbased navigation systems. This paper presents the first attempt to investigate how to effectively utilize vehicles' trajectory information in a privacy-preserving manner. In our design, the trajectory information is combined with the traffic statistics to improve the performance of data forwarding in road networks. Through theoretical analysis and extensive simulation, it is shown that our design outperforms the existing scheme.
\end{abstract}

\section{Introduction}

With the standardization of Dedicated Short Range Communication (DSRC) by IEEE [1], Vehicular Ad Hoc Networks (VANETs) have recently reemerged as one of promising research areas for safety and connectivity in road networks. Currently, most research and development fall into one of two categories: (i) vehicle-to-vehicle (v2v) communications [2] and (ii) vehicle-to-infrastructure (v2i) communications [3][5]. In the meantime, the GPS technology has been adopted for navigation purposes at an unprecedented rate. It is expected that approximately 300 million GPS devices will be shipped in 2009 alone [6]. It becomes a very timely topic to develop novel applications by integrating the cutting-edge DSRC and GPS technologies.

Specifically, this work is motivated by the observed trend that a large number of vehicles have started to install GPSreceivers for navigation and the drivers are guided by these GPS-based navigation systems to select better driving paths in terms of the physically shortest path or the vehicular lowdensity traffic path. Therefore, the nature research question is how to make the most of this trend to improve the performance of vehicular ad hoc networks.

Let's consider the scenario where Internet access points are sparsely deployed along the roadways for the road-side reports, such as the time-critical reports of driving accident or driving hazard. The Internet access points have limited communication coverage, so the vehicles cannot directly transmit their packets to the Internet access points. To support such a scenario, the carry-and-forward techniques are proposed for use by several opportunistic forwarding schemes [3], [7]. In these schemes, vehicles carry or forward packets progressively close to an access point by selecting potential shortest path based on traffic statistics. Without considering individual vehicles' trajectories, these forwarding schemes can be inefficient, especially in light-traffic road networks (e.g., rural-area road networks). This is because that the probability to forward packets to other vehicles at intersections is low in light-traffic road networks and it would be the case that vehicles carry packets towards the wrong direction, introducing excessive long delays.

This paper, for the first time, proposes a data forwarding scheme utilizing the vehicles' trajectory information for lighttraffic road networks. The first challenge is how to use the trajectory information in a privacy-preserving manner, while improving the data forwarding performance. To resolve this challenge, we design a local algorithm to compute expected data delivery delay (EDD) at individual vehicles to an access point, using private trajectory information and known traffic statistics. Only the computed delay is shared with neighboring vehicles. The vehicle with the shortest EDD is selected as the next packet carrier for its neighboring vehicles. The other challenge is how to model an accurate road link delay, a delay defined as the time taken for a packet to travel through a road segment using carry-and-forward. To resolve this challenge, we accurately model road link delay, based on traffic density information obtained from the GPS-based navigation system. Our intellectual contributions are as follows:

- An analytical link delay model for packet delivery along a road segment that is much more accurate than that of the state-of-the-art solution.

- An expected E2E delivery delay computation based on individual vehicle trajectory for the better decision making on the packet forwarding.

The rest of this paper is organized as follows: Section 2 describes the problem formulation. Section 3 describes our link delay model. Section 4 explains the design of the trajectorybased forwarding including the computation of the end-to-end delivery delay. Section 5 evaluates our design. We summarize related work in Section 6 and conclude this paper in Section 7. 


\section{Problem Formulation}

Given a road network with an Internet access point, the research problem is to minimize the end-to-end delivery delay of packets to the Internet access point. In this paper, we focus on one-way data delivery which is useful for the time-critical reports, such as vehicle accidents, road surface monitoring and driving hazards. We leave two-way delivery as future work. In this paper, we refer (i) Vehicle trajectory as the moving path from the vehicle's starting position to its destination position in a road network; (ii) Expected Delivery Delay (EDD) as the expected time taken to deliver a packet generated by a vehicle to an Internet access point via the VANET; (iii) Carry delay as a part of the delivery delay introduced while a packet is carried by a moving vehicle; (iv) Communication delay as a part of the delivery delay introduced while a packet is forwarded among vehicles. Our work is based on the following four assumptions:

- The geographical location information of packet destinations, such as Internet access points (APs), is available to vehicles. A couple of studies have been done to utilize the Internet access points available on the road-sides [5].

- Vehicles participating in VANET have a wireless communication device, such as the Dedicated Short Range Communications (DSRC) device [1]. Nowadays many vehicle vendors, such as GM and Toyota, are planning to install DSRC devices at vehicles [8].

- Vehicles are installed with a GPS-based navigation system and digital road maps. Traffic statistics, such as vehicle arrival rate $\lambda$ and average vehicle speed $v$ per road segment, are available via a commercial navigation service, such as Garmin Traffic [9].

- Vehicles know their trajectory by themselves. However, vehicles do not release their trajectory to other vehicles for privacy concerns.

It should be noted that in the VANET scenarios, the carry delay is several orders-of-magnitude longer than the communication delay. For example, a vehicle takes 90 seconds to travel along a road segment of 1 mile with a speed of $40 \mathrm{MPH}$, however, it takes only ten of milliseconds ${ }^{1}$ to forward a packet over the same road segment, even after considering the retransmission due to wireless link noise or packet collision. Therefore, since the carry delay is the dominating part of the total delivery delay, in the rest of the paper we focus on the carry delay for the sake of clarity, although the small communication delay does exist in our design. In the next sections, we will explain our Link delay modeling and Trajectory-based data forwarding.

\section{The Link Delay Model}

This section analyzes the link delay for one road segment with one-way vehicular traffic given the vehicle inter-arrival time, the vehicle speed and the communication range. We

1. Note that the data rate in DSRC [1] is from 6 27 Mbps and transmission range can extend to almost 1,000 meters.

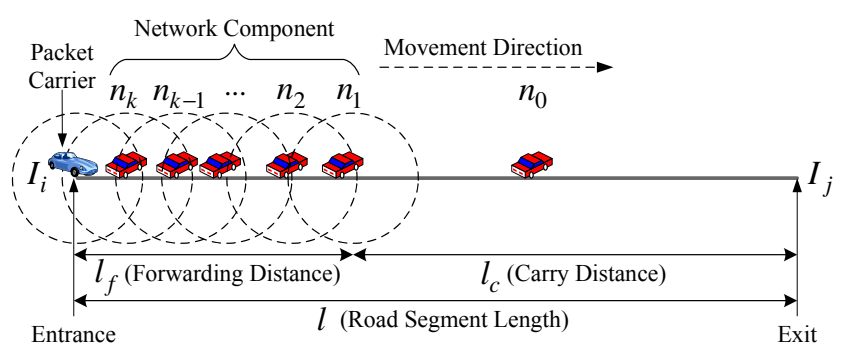

Figure 1. Forwarding Distance $l_{f}$ and Carry Distance $l_{c}$

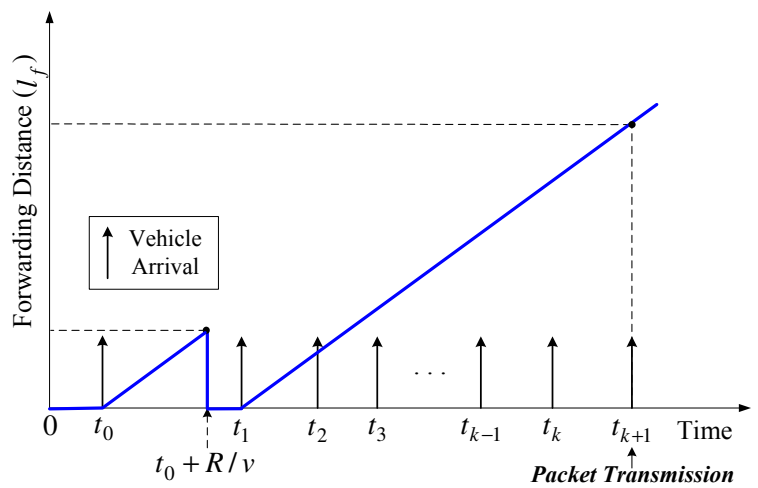

(a) Forwarding Distance $\left(l_{f}\right)$ over Time

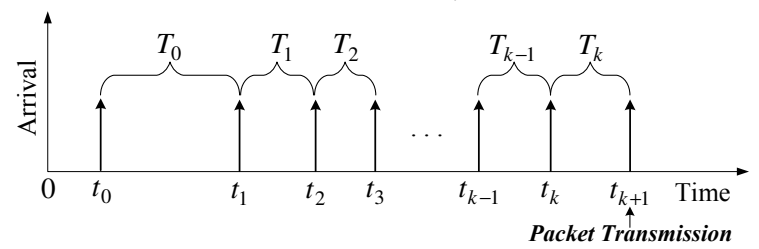

(b) Vehicle Arrival Sequence on One-way Road Segment

Figure 2. Forwarding Distance $\left(l_{f}\right)$ for Vehicle Arrivals

leave the link delay for a two-way road segment as future work. Three terms for the link delay model are defined as follows:

Definition 1 (Connected Component): Let Connected Component be a group of vehicles that can communicate with each other via either one-hop or multi-hop communication. Figure 1 shows a connected component consisting of vehicles $n_{1}, \ldots, n_{k}$.

Definition 2 (Forwarding Distance): Let Forwarding Distance (denoted as $l_{f}$ ) be the physical distance a packet travels via wireless communication within a road segment starting from the entrance. Figure 1 shows the forwarding distance $l_{f}$ for the connected component.

Definition 3 (Carry Distance): Let Carry Distance (denoted as $l_{c}$ ) be the physical distance a packet is carried by a vehicle within a road segment. Figure 1 shows the carry distance $l_{c}$ of vehicle $n_{1}$.

Let $v$ be the vehicle speed. By ignoring the small communication delay, the link delay $d_{i j}$ along a road with the length of $l$ is the corresponding carry delay. We have,

$$
d_{i j}=\frac{l_{c}}{v} \quad \text { where } l_{c}=l-l_{f} .
$$

Therefore, the expected link delay $E\left[d_{i j}\right]$ is:

$$
E\left[d_{i j}\right]=\left(l-E\left[l_{f}\right]\right) / v .
$$


In Equation 2, in order to obtain the expected link delay $E\left[d_{i j}\right]$, we need to derive the expected forwarding distance $E\left[l_{f}\right]$ first. Clearly the forwarding distance $l_{f}$ equals the communication length of the connected component that is near the entrance as shown in Figure 1. To illustrate our modeling approach, we use Figure 2(a) to explain how the forwarding distance $l_{f}$ change over time under different traffic arrival patterns.

- At time $t_{0}$, vehicle $n_{0}$ arrives. Since $n_{0}$ moves at the constant speed $v$, the forwarding distance $l_{f}$ increases linearly at the rate of $v$. During the time interval $\left[t_{0}, t_{0}+\right.$ $R / v]$, no other vehicle arrives, forcing $n_{0}$ to move out of the communication range of $I_{i}$. As a result, $l_{f}$ reduces to zero after $t_{0}+R / v$.

- At time $t_{1}$, vehicle $n_{1}$ arrives. Similarly, the forwarding distance $l_{f}$ increases linearly at the rate of $v$. In this case, vehicles $n_{2}, \ldots, n_{k}$ arrive at $I_{i}$ with the inter-arrival time less than $R / v$, forming a connected component of $k$ vehicles.

To formally derive $E\left[l_{f}\right]$, we model the forwarding distance $l_{f}$ as the sum of the inter-vehicle distance of vehicles within the component at any time. Figure 2(b) shows the corresponding vehicle arrival times as in Figure 2(a). Let $t_{h}$ be the arrival time of the $h$-th vehicle. Let $T_{h}$ be the inter-arrival interval of the $h$-th vehicle and the $(h+1)$-th vehicle. $T_{h}$ is assumed to be an exponential random variable with arrival rate $\lambda$. This assumption has been shown valid in [10], because the Kolmogorov-Smirnov test can accurately approximate the statistics of vehicle inter-arrival time based on the empirical data for a real roadway into an exponential distribution.

As shown in Figure 2(b), when the vehicle $n_{k+1}$ carrier arrives at $t_{k+1}$ with an outgoing packet, the forwarding distance $l_{f}$ is zero if $T_{k}=t_{k+1}-t_{k}>R / v$, otherwise $l_{f}$ is the communication length of the connected component $\sum_{h=1}^{k} T_{h} v$ if $T_{k}=t_{k+1}-t_{k}<R / v$. We note the expected number of vehicle inter-distances (i.e., $v T_{h}$ ) within a connected component is the ratio between $P\left[v T_{h} \leq R\right]$ and $P\left[v T_{h}>R\right]$, according to detailed derivation in our technical report [11] for the link delay modeling. Therefore, we obtain $E\left[l_{f}\right]$ for the road segment $\left(I_{i}, I_{j}\right)$ as follows:

$$
E\left[l_{f}\right]=E\left[v T_{h} \mid v T_{h} \leq R\right] \times \frac{P\left[v T_{h} \leq R\right]}{P\left[v T_{h}>R\right]}
$$

From (3), we can see that $E\left[l_{f}\right]$ is the multiplication of (i) the average inter-distance of two adjacent vehicles within the same component and (ii) the ratio of the probability that the inter-distance is not greater than the communication range to the probability that the inter-distance is greater than the communication range. As the inter-arrival time decreases, this ratio increases, leading to the longer average forwarding distance; note that as the inter-arrival time decreases, the average inter-distance decreases, but the increasing rate of the ratio is much faster. Therefore, this fits well our intuition that the shorter inter-arrival time, the shorter inter-distance for communication, leading to the longer average forwarding distance.

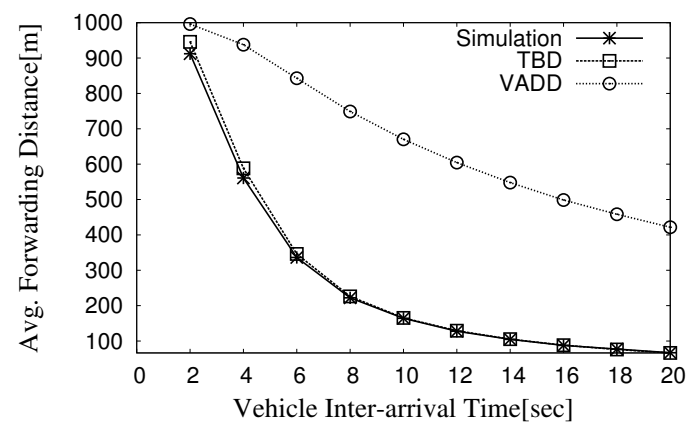

Figure 3. Validation and Comparison of Analytical Models

Figure 3 shows the average forwarding distance $l_{f}$ comparison among simulation model and two analytical models for one-way roadway: (i) Our $T B D$ link model [11] and (ii) VADD link model proposed by Zhao and Cao [3]. As shown in Figure 3, our link model gives very accurate average forwarding distance $l_{f}$ estimates under different inter-arrival intervals. The reason $V A D D$ is not accurate is that $V A D D$ considers the sum of the lengths of all connected vehicles, while missing the fact that only the connected component starting from the entrance can actually be used for data forwarding.

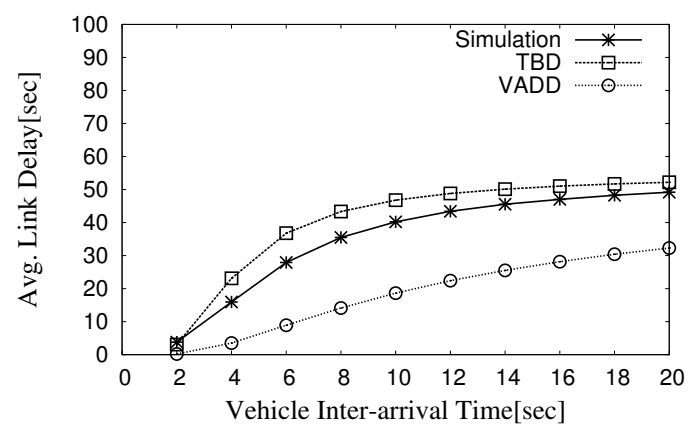

Figure 4. Link Delay Comparison for Model Validation

The above modeling process assumes the speed $v$ of vehicles is constant. Clearly it does not hold well in practice, because for four-lane roadways, the vehicle speed deviation is 6.2 MPH (i.e., $9.98 \mathrm{~km} / \mathrm{h}$ ), according to field study conducted by Victor Muchuruza [12]. To investigate how robust our link delay model is, we test the accuracy of our model under a realistic setting where the vehicle speed follows a normal distribution of $N(40,7) \mathrm{MPH}$. Our model is compared with simulation, which approximates the ground truth, and VADD [3]. Figure 4 illustrates that as the vehicle speed deviation is within the realistic bound, the link delay of $T B D$ is closer to the simulation result than that of $V A D D$.

\section{TBD: E2E Delay Model and Protocol}

In this section, we explain the design of our trajectorybased forwarding with two steps: We will first explain how to compute the Expected Delivery Delay (EDD) considering both vehicular traffic statistics and individual vehicle trajectory in section 4.1 and then describe how vehicles perform the data forwarding based on EDD in section 4.2. 


\subsection{End-to-End Delay Model}

In this section, we model the EDD with a stochastic model [3] for a given road network. We define the road network graph for the EDD computation as follows:

Definition 4 (Road Network Graph): Let a road network graph be the directed graph of $G=(V, E)$, where $V=$ $\left\{v_{1}, v_{2}, \ldots, v_{n}\right\}$ is a set of intersections in the road network and $E=\left[e_{i j}\right]$ is a matrix of edge $e_{i j}$ for vertices $v_{i}$ and $v_{j}$ such that $e_{i j} \neq e_{j i}$. Figure 5 shows a road network graph.

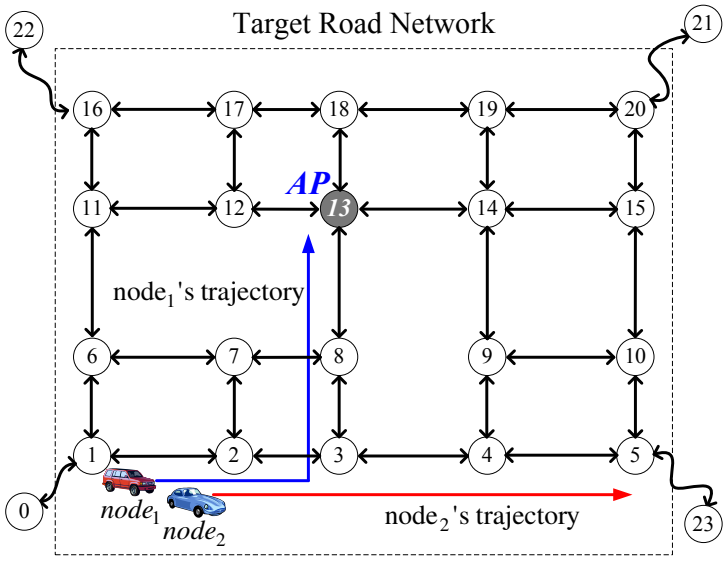

Figure 5. Road Network Graph for Data Forwarding

To estimate end-to-end delay, we cannot use the traditional shortest path algorithms, such as Dijkstra's shortest path algorithm. This is because when the packet carrier arrives at an intersection, it is not guaranteed that it can meet another vehicle moving towards the most preferred direction. In this case, the packet carrier needs to determine whether it can forward its packet to another vehicle moving towards other preferred directions or has to carry it with itself to the next intersection on its trajectory. In order to consider all of the possible cases in the forwarding at each intersection, we formulate the data delivery based on this carry-and-forward as the stochastic model.

4.1.1. Expected Delivery Delay at Intersection. In this section, we explain how to compute the EDD at an intersection, using a stochastic model. Suppose that a packet at intersection $i$ is delivered towards intersection $j$. Let $d_{i j}$ be the link delay for edge $e_{i j}$ in Equation 1. We note the expected delay EDD at an intersection depends on the forwarding direction (i.e., edge). Therefore, we use $D_{i j}$ denote the EDD at the intersection $i$ when the edge $e_{i j}$ is used as the forwarding edge. We formulate $D_{i j}$ recursively as follows:

$$
\begin{aligned}
D_{i j} & =d_{i j}+E[\text { delivery delay at } j \text { by forwarding or carry }] \\
& =d_{i j}+\sum_{k \in N(j)} P_{j k} D_{j k}
\end{aligned}
$$

where $N(j)$ is the set of neighboring intersections of intersection $j$. We use this stochastic model to compute the EDD at intersection $i$ because the packet will be delivered with some probability to one of outgoing edges at intersection $j$. This means that when the carrier of this packet arrives at intersection $j$, the next carrier on each outgoing edge towards intersection $k$ will be met with probability $P_{j k}$. We will explain how to compute the probability $P_{j k}$ later.

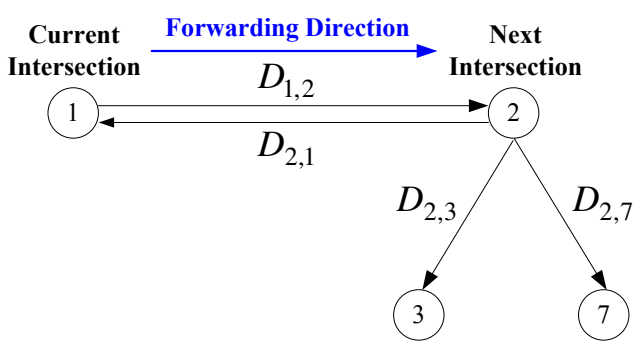

Figure 6. EDD Computation for Edge $e_{1,2}$

For example, suppose that as shown in Figure 6, a packet carried by a vehicle arrives at intersection 1 and is sent towards intersection 2. The EDD of $D_{1,2}$ denotes the endto-end delivery delay when the carrier sends its packet to the AP via the edge $e_{1,2}$. First, it will take $d_{1,2}$ seconds to deliver a packet to the intersection 2 via $e_{1,2}$. Once the packet arrives at intersection 2 , there are three possible cases to deliver the packet. In other words, the packet can be forwarded to one of three neighboring intersections (i.e., intersection 1,3 or 7 ) of intersection 2 with some probability. Let $D_{2,1}, D_{2,3}$ and $D_{2,7}$ be the EDDs for three edges $e_{2,1}, e_{2,3}$ and $e_{2,7}$, respectively. We can compute $D_{1,2}$ using the stochastic model in (4) as follows:

$$
D_{1,2}=d_{1,2}+P_{2,1} D_{2,1}+P_{2,3} D_{2,3}+P_{2,7} D_{2,7} .
$$

Let $n$ be the number of directed edges in the road network graph $G=(V, E)$, as shown in Figure 5. We have $n$ variables of $D_{i j}$ for directed edge $e_{i j} \in E(G)$. Since we have $n$ variables and $n$ linear equations of (4), we can solve this linear system using the Gaussian Elimination algorithm.

We start to explain how to compute the probability $P_{j k}$ in (4). $P_{i j}$ is defined as the average forwarding probability that a packet at intersection $i$ will be delivered to a vehicle moving towards the neighboring intersection $j$.

Contact Probability: Contact Probability is defined as the chance that a vehicle can encounter another vehicle at an intersection. Let $R$ be communication range. Let $v$ be the mean vehicle speed in the intersection area of intersection $i$ which is a circle of radius $R$. Let $T_{i}$ be the duration during which a vehicle is able to communicate with the vehicles around the intersection $i$. Clearly, $T_{i}$ is affected by the vehicle speed, the communication range, the traffic signal pattern and the queueing delay. In practice, average $T_{i}$ can be obtained through empirical measurements. In this study, we use a simplifying model to calculate $T_{i}$ by assuming the nominal communication range is $R$ and a constant speed is $v$. Therefore, $T_{i}=2 R / v$. We note our design can use empirical $T_{i}$ measurements if available. Let $C P_{i j}$ be the contact probability that a packet carrier in the intersection area of $i$ will meet at least one vehicle moving towards $j$ for during $T_{i}$. Suppose that the vehicle arrival at the directed edge $e_{i j}$ is 
Poisson process with vehicle arrival rate $\lambda_{i j}$. Thus, $C P_{i j}$ is computed using the Poisson Process probability as follows:

$$
C P_{i j}=1-e^{-\lambda_{i j} T_{i}} .
$$

Forwarding Probability: At an intersection, forwarding is probabilistic in nature, therefore a packet is forwarded with best-effort. Let's define the forwarding probability as the chance that a packet carrier at intersection $i$ can forward a packet to another vehicle moving towards one of the neighboring intersections $j_{k}$ for $k=1 . . m$. We note there is a clear distinction between the contact probability and forwarding probability, because a packet will not be forwarded to a contacted vehicle that moves to a wrong direction.

To calculate forwarding probability, we need to sort edges based on the forward priority. For an intersection $i$ with $m$ forwarding edges $e_{i j_{k}}(k=1 \ldots m)$, we can sort them in nondecreasing order, based on their geographically shortest path length from intersection $i$ to a packet destination (i.e., $A P$ ) via the edge $e_{i j_{k}}$. This heuristic is based on the observation that the edge on the geographically shortest path tends to provide the shortest delivery path; note that the intersection model of [3] uses the angle between the packet destination and the edge for the enumeration, but the smallest angle does not always give the shortest path in the road networks of nongrid topology. Therefore, the forwarding probability $P_{i j_{k}}^{\prime}$ for each edge $e_{i j_{k}}$ is computed as follows:

$$
P_{i j_{k}}^{\prime}= \begin{cases}C P_{i j_{1}} & \text { for } k=1, \\ \left(\prod_{s=1}^{k-1}\left(1-C P_{i j_{s}}\right)\right) C P_{i j_{k}} & \text { for } k=2 . . m .\end{cases}
$$

Conditional Forwarding Probability: Clearly, a packet should not be forwarded to the edge that is worse than the edge the carrier moves toward, therefore, we need to compute the conditional forwarding probability that a packet carrier moving on edge $e_{i j_{h}}$ can forward its packet to another vehicle moving on $e_{i j_{k}}$, that is, $P_{i j_{k} \mid i j_{h}}=$ $P$ [packet is forwarded to $e_{i j_{k}} \mid$ carrier moves on $\left.e_{i j_{h}}\right]$. The conditional forwarding probability $P_{i j_{k} \mid i j_{h}}$ is computed as follows:

$$
P_{i j_{k} \mid i j_{h}}= \begin{cases}P_{i j_{k}}^{\prime} & \text { for } k<h, \\ 1-\sum_{s=1}^{k-1} P_{i j_{s}}^{\prime} & \text { for } k=h, \\ 0 & \text { for } k>h .\end{cases}
$$

Average Forwarding Probability: Finally, we can compute the average forwarding probability $P_{i j_{k}}$ that a packet arriving at intersection $i$ will be delivered to the neighboring intersection $j_{k}$ by either forwarding or carry. In order to compute $P_{i j_{k}}$ for the packet-delivered intersection $j_{k}$, we need the branch probability $B_{i j_{h}}$ that a packet carrier arriving at intersection $i$ will move to intersection $j_{h}$ for $j_{h} \in N(i)$. This branch probability can be obtained from the vehicular traffic statistics on the edge $e_{i j_{h}}$. Therefore, $P_{i j_{k}}$ is calculated as follows:

$$
P_{i j_{k}}=\sum_{j_{h} \in N(i)} B_{i j_{h}} P_{i j_{k} \mid i j_{h}}
$$

For example, as shown in Figure 7, suppose that a packet carrier is placed at intersection 2 in Figure 5 and moves to

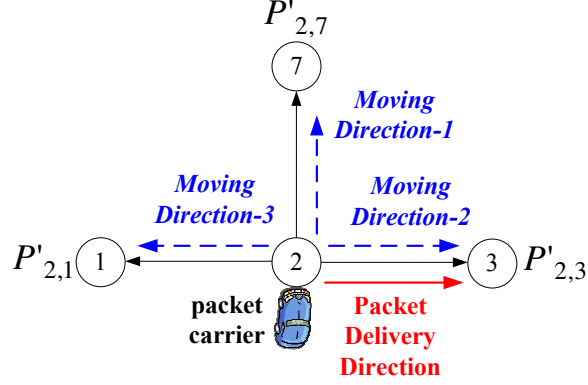

Figure 7. Average Forwarding Probability $P_{2,3}$

one of the neighboring intersections with the corresponding branch probability $B_{2, j}$ for $j=\{1,3,7\}$; that is, there are three directions for the packet carrier to take, such as Moving Direction-1, Moving Direction-2 and Moving Direction-3. We want to compute the average forwarding probability $P_{2,3}$ that the packet carrier will deliver its packet onto edge $e_{2,3}$. We assume that the ascending order of the shortest path length from intersection 2 towards the AP via the three edges is $e_{2,7}$, $e_{2,3}$ and $e_{2,1}$. According to this assumption, the contacting order for packet forwarding is the same (i.e., $e_{2,7}, e_{2,3}$ and $\left.e_{2,1}\right)$ and the forwarding probabilities for these three edges are $P_{2,7}^{\prime}, P_{2,3}^{\prime}$ and $P_{2,1}^{\prime}$, respectively. Therefore, the average forwarding probability $P_{2,3}$ is computed from (8) as follows:

$$
\begin{aligned}
P_{2,3} & =B_{2,1} P_{2,3 \mid 2,1}+B_{2,3} P_{2,3 \mid 2,3}+B_{2,7} P_{2,3 \mid 2,7} \\
& =B_{2,1} P_{2,3}^{\prime}+B_{2,3}\left(1-P_{2,7}^{\prime}\right) .
\end{aligned}
$$

Note that (a) $P_{2,3 \mid 2,1}=P_{2,3}^{\prime}$ since the shortest path length for the carrier's moving edge $e_{2,1}$ is longer than that for the forwarding edge $e_{2,3}$, so the carrier tries to forward its packets onto $e_{2,3}$; (b) $P_{2,3 \mid 2,3}=1-P_{2,7}^{\prime}$ since the shortest path length for the edge $e_{2,7}$ has the shortest among the three edges; (c) $P_{2,3 \mid 2,7}=0$ since the shortest path length for the carrier's moving edge $e_{2,7}$ is shorter than that for the forwarding edge $e_{2,3}$, so the carrier does not try to forward its packets onto $e_{2,3}$.

We note this EDD model computes $D_{i j}$ without considering the trajectory. For example, if two vehicles node $_{1}$ and node $_{2}$ are placed at the same intersection 1 in Figure 5, their EDDs towards the same packet-delivered edge $e_{1,2}$ are the same with each other. Therefore, only with this intersection EDD model, the individual vehicle's trajectory does not affect the computation of EDD, so we cannot determine to choose which one as the best next carrier. In the next section, we explain how the vehicle trajectory can be added to the EDD computation.

4.1.2. Expected Delivery Delay based on Trajectory. In this section, we explain how to compute the expected E2E delivery delay (EDD) based on the vehicle trajectory. A trajectory is defined as the moving path from a vehicle's starting position to its destination position in a road network;

The main idea of trajectory-based forwarding is to divide the delivery process recursively into two steps: (i) The packet carry process at the current vehicle and (ii) the delivery process after the packet leaves this vehicle. In the case of light traffic, it is possible that a vehicle could carry a packet continuously over multiple edges. 
Suppose the packet is with the current vehicle. This vehicle will travel along a trajectory denoted by a sequence of intersections: $1 \rightarrow 2 \rightarrow \cdots \rightarrow M$. Let $C_{i j}$ be the total time taken to carry the packet by the vehicle from the intersection $i$ to the intersection $j$ along the trajectory $(1 \leq i \leq j \leq M)$. Formally, $C_{i j}=\sum_{k=i}^{j-1} l_{k, k+1} / v$. As a reminder, $P_{m n}^{\prime}$ is the forwarding probability in (6) that the vehicle at intersection $m$ can forward its packets to another vehicle moving towards the neighboring intersection $n$. We denote $P_{m n}^{c}$ as the carry probability that the vehicle cannot forward its packet at intersection $m$, and so has to carry its packets to the adjacent intersection $n$. Formally, $P_{m n}^{c}=1-\prod_{k \in N(m)} P_{m k}^{\prime}$. The expected end-to-end delay $D$ at the vehicle is computed as follows:

$$
\begin{aligned}
D= & \sum_{j=1}^{M}(P[\text { a packet is carried from intersection } 1 \text { to } j] \\
& \left.\times\left(C_{1 j}+E[\text { delivery delay at intersection } j]\right)\right) \\
= & \sum_{j=1}^{M}\left(\left(\prod_{h=1}^{j-1} P_{h, h+1}^{c}\right) \times\left(C_{1 j}+\sum_{k \in N(j)} P_{j k}^{\prime} D_{j k}\right)\right)
\end{aligned}
$$

In (9), $P$ [a packet is carried from intersection 1 to $j]=$ $\prod_{h=1}^{j-1} P_{h, h+1}^{c}$ is the carry probability along the trajectory from intersection 1 to the intersection $j$. $E[$ delivery delay at intersection $j]=\sum_{k \in N(j)} P_{j k}^{\prime} D_{j k}$ is the EDD after the packet leaves the current vehicle at $j$.

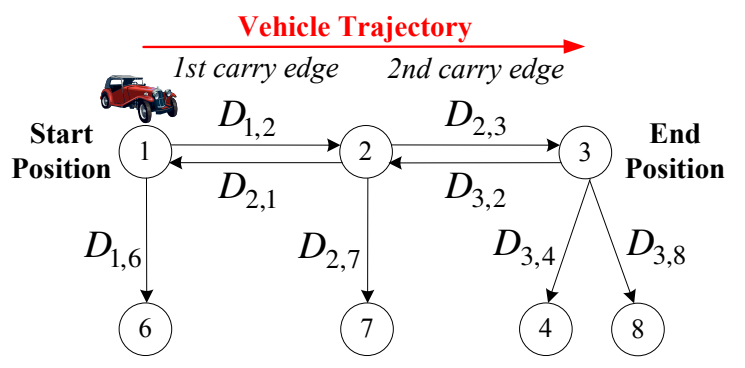

Figure 8. EDD Computation for Vehicle Trajectory

For example, as shown in Figure 8, let the trajectory be $1 \rightarrow 2 \rightarrow 3$ in the road network in Figure 5. First, the vehicle at intersection 1 can try to forward the packets to the neighboring intersections 2 and 6 . If it cannot forward the packets at the intersection 1 , it must carry them by the next intersection 2. When it arrives at intersection 2 , it can try to forward again. If it cannot forward again, it will carry the packet to the third intersection 3. At the destination, if the vehicle cannot forward, it discards the packets. With this scenario, the expected delivery delay $D$ is computed as follows:

$$
\begin{aligned}
D= & P_{1,2}^{\prime} D_{1,2}+P_{1,6}^{\prime} D_{1,6}+P_{1,2}^{c}\left(C_{1,2}+P_{2,1}^{\prime} D_{2,1}\right. \\
& \left.+P_{2,3}^{\prime} D_{2,3}+P_{2,7}^{\prime} D_{2,7}\right)+P_{1,2}^{c} P_{2,3}^{c}\left(C_{1,3}\right. \\
& \left.+P_{3,2}^{\prime} D_{3,2}+P_{3,4}^{\prime} D_{3,4}+P_{3,8}^{\prime} D_{3,8}\right) .
\end{aligned}
$$

So far, we have explained how to compute the EDD based on the vehicular traffic statistics and individual vehicle trajectory. In the next section, we will explain how vehicles can use their EDDs in the packet forwarding process.

\subsection{Forwarding Protocol Design}

Now we will briefly explain how vehicles can use their EDDs in the data forwarding to deliver data packets to the destination in the given road network. Our TBD forwarding rule is as simple as the following:

Within a connected component, packets are forward to the vehicle with a minimum EDD.

Each individual vehicle updates its $E D D$ with (9), based on its trajectory from the current position to the destination position every update period (e.g., one second). This vehicle's EDD is broadcasted within the connected component. In this way, each vehicle can recognize the EDDs of other vehicles. Besides using this simple broadcast method, we can apply more advanced group management protocols for ad-hoc networks such as in [13], which handles group update, mergence and partition in a more efficient manner. We leave this type of optimization as future work, because in vehicular networks, communication energy is not a key resource constraint.

\section{Performance Evaluation}

In this section, we evaluate the performance of $T B D$ by comparing it with a state-of-the-art scheme, VADD (using the link delay model and the Direction-First-Probe forwarding protocol proposed in [3]). The evaluation is based on the following:

- Performance Metric: We use average delivery delay as performance metric.

- Parameters: We investigate the impact of (i) vehicular traffic density, (ii) vehicle speed, and (iii) vehicle speed deviation.

A road network with 36 intersections is used in the simulation and one Internet access point is deployed in the center of the network. Each vehicle's movement pattern is determined by a random waypoint model where the vehicle moves along the shortest path from a randomly selected source position to a randomly selected destination position. During the simulation, following an exponential distribution with a mean of 5 seconds, packets are dynamically generated from 10 vehicles in the road network. The total number of generated packets is 50,000 and the simulation is continued until all of these packets are either delivered or dropped due to TTL expiration. The system parameters are selected based on a typical DSRC scenario [1]. Unless otherwise specified, the default values in Table 1 are used.

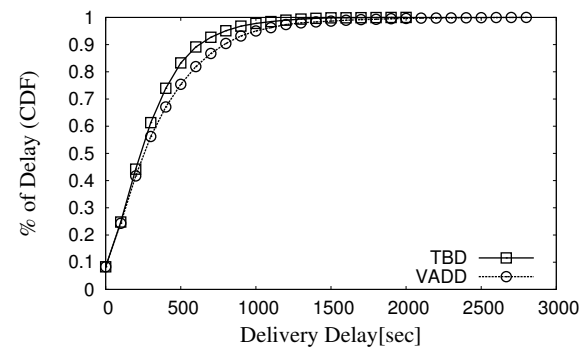

Figure 9. CDF Comparison for Delivery Delay 


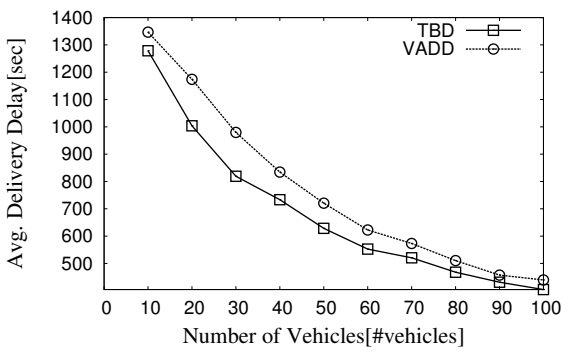

(a) Impact of the Number of Vehicles

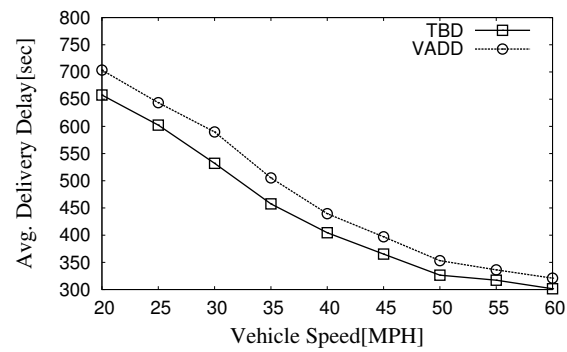

(b) Impact of Vehicle Speed

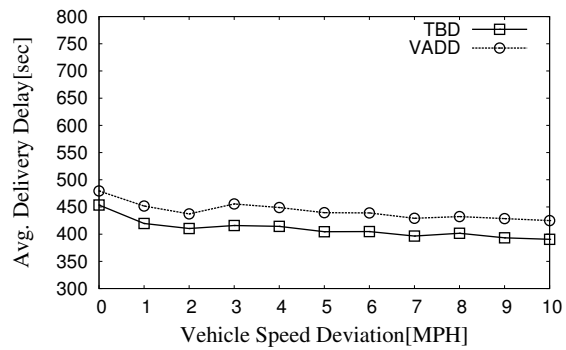

(c) Impact of Vehicle Speed Deviation

Figure 10. Performance Comparison between TBD and VADD

Table 1. Simulation Configuration

\begin{tabular}{|c|l|}
\hline Parameter & Description \\
\hline Road network & $\begin{array}{l}\text { The number of intersections is 36. The area } \\
\text { of the road map is } 4.2 \text { miles } \times 3.7 \text { miles. }\end{array}$ \\
\hline Communication range & $R=200$ meters (i.e., 656 feet). \\
\hline Number of vehicles & $\begin{array}{l}\text { The number } N \text { of vehicles moving within } \\
\text { the road network. The default of } N \text { is } 100 .\end{array}$ \\
\hline Time-To-Live & $\begin{array}{l}\text { The expiration time of a packet. The default } \\
\text { of } T T L \text { is } \infty \text { (i.e., no timeout). }\end{array}$ \\
\hline Vehicle speed & $\begin{array}{l}v \sim N\left(\mu_{v}, \sigma_{v}\right) \text { where } \mu_{v}=\{20,25, \ldots, 60\} \\
\text { MPH and } \sigma_{v}=\{0,1, \ldots, 10\} \text { MPH. The } \\
\text { default of }\left(\mu_{v}, \sigma_{v}\right) \text { is }(40,5) .\end{array}$ \\
\hline
\end{tabular}

\subsection{Forwarding Behavior Comparison}

We compare the forwarding behaviors of $T B D$ and $V A D D$ with the cumulative distribution function (CDF) of the actual packet delivery delays. From Figure 9, it is very clear that $T B D$ has smaller packet delivery delay than $V A D D$. For any given packet deliver delay, TBD always has a larger CDF value than $V A D D$ before they both reach $100 \% \mathrm{CDF}$. For example, $T B D$ reaches $90 \%$ CDF with a delivery delay of about 600 seconds while the value for $V A D D$ is about 800 seconds. In other words, on average, the packet delivery delay for $T B D$ is smaller than that for $V A D D$ and we will show this quantitatively in the following subsections.

\subsection{The Impact of Vehicle Number $N$}

The number of vehicles in the road network determines the vehicular traffic density in a road network. In this subsection, we intend to study how effectively $T B D$ can forward packets towards the access point using individual vehicles' trajectory information. Through our extensive simulations, we observe that under low vehicular traffic density, TBD significantly outperforms $V A D D$ in terms of packet delivery delay.

Figure 10(a) shows the packet delivery delay comparison between $T B D$ and $V A D D$ with varying number of vehicles under low vehicular traffic density. As shown in Figure 10(a), $T B D$ has smaller packet delivery delay than $V A D D$ at all vehicular densities. The smallest delay reduction is $5 \%$ at $N=10$ while the largest delay reduction is $16.3 \%$ at $N=30$. This shows that in the extremely sparse road networks, such as $N=10$, the trajectory in $T B D$ has less contribution than in the cases of not-so-sparse road networks, such as $N \geq 20$.
This is because when the number of vehicles is so small, the probability that vehicles can meet each other is low and also the probability that the carriers will pass the access point is low. However, in the sparse road networks, by using both the trajectory and the vehicular traffic statistics, TBD has an average of $10.3 \%$ delivery delay reduction (from $N=10$ to $N=100$ ) over $V A D D$, which only considers the vehiclular traffic statistics.

As a result, we found that $T B D$ not only provides significant better data forwarding quality than $V A D D$ in light-traffic road networks which is targeted in this paper, but also has smaller packet delivery delay even at high-traffic conditions.

\subsection{The Impact of Vehicle Speed $\mu_{v}$}

In this subsection, we are interested to investigate how the change of mean vehicle speed affects the delivery delay. Figure 10(b) shows the delivery delay under different mean vehicle speeds. As shown in the Figure 10(b), for both $T B D$ and $V A D D$, the higher vehicle speed leads to the shorter delivery delay for both $T B D$ and $V A D D$. This is because the high vehicle speed yields high vehicle arrival rate at each road segment, leading to the shorter delivery delay. However, at all vehicle speeds, the $T B D$ still outperforms $V A D D$.

\subsection{The Impact of Vehicle Speed Deviation $\sigma_{v}$}

The vehicles moving with a high speed deviation can construct a longer ad-hoc network component for communications, so the delivery delay in a high speed deviation can be shorter than that in a low speed deviation. This is because in such a high speed deviation, fast moving vehicles can connect two isolated network components with the communication range when they pass the middle of the two isolated components. On the other hand, in a low speed deviation, such as zero deviation, if two isolated components are isolated from the communication, they cannot be merged into a longer component.

Figure 10(c) illustrates this observation for the delivery delay in the vehicle speed deviation. The higher vehicle speed deviation overall tends to lead to the slightly shorter delivery delay in both $T B D$ and $V A D D$. 


\section{Related Work}

Data forwarding and data access issues in VANET have gained a lot of attentions recently [3]-[5], [14]. The data forwarding in VANET is different from that in the traditional mobile ad-hoc networks (MANETs) for the reason of (i) vehicles are moving on the physically constrained areas (i.e., roadways) and (ii) the communication shortest path does not always match the physical shortest path due to heterogeneous vehicular traffic conditions on road segments. These unique characteristics of the road networks open the doors of research opportunities for the data forwarding in the VANET. Also, the frequent network partition and mergence due to the high mobility makes the MANET routing protocols based on endto-end connectivity ineffective in the VANET settings [10]. Thus, in order to deal with this frequent network partition and mergence, the carry-and-forward approaches are necessary.

Recently, these carry-and-forward data forwarding schemes have been proposed, investigating the layout of road networks [3], [4], [14]. VADD [3] proposes the data forwarding using a stochastic model based on vehicular traffic statistics in order to achieve the lowest delivery delay from mobile vehicles to a stationary packet destination. On the other hand, Delay-Bounded Routing [4] proposes data forwarding schemes to minimize the channel utilization, satisfying the user-defined delay bound. Our TBD, in contrast, improves forwarding performance by utilizing the vehicle trajectory information along with vehicular traffic statistics in order to compute the accurate expected delivery delay for better forwarding decision making. $M D D V$ [14] proposes a forwarding scheme in VANET to allow the predefined packet trajectory. This packet trajectory is the path through which the packet traverses, which is different from the vehicle trajectory in TBD. Cabernet [5] proposes one-hop Internet access schemes using open $\mathrm{WiFi}$ access points in vehicular networks.

\section{Conclusion}

In this paper, we propose a trajectory-based data forwarding scheme for light-traffic road networks, where the carry delay is the dominating factor for the end-to-end delivery delay. We compute the aggregated end-to-end carry delay using the individual vehicle trajectory along with the vehicular traffic statistics. Our design allows vehicles to share their trajectory information without exposing their actual trajectory to neighbor vehicles. This privacy-preserving trajectory sharing scheme is made possible by exchanging only the expected delay value using local vehicle trajectory information. We also propose a link delay model based on the common assumption of exponential vehicle inter-arrival time. It is shown to be more accurate than the state-of-the-art solution. With the increasing popularity of vehicular ad-hoc networking, we believe that our forwarding scheme opens a first door for exploiting the potential benefit of the vehicle trajectory for the performance of VANET networking. As future work, we will explore indepth research on the data forwarding from Internet access points to moving vehicles for supporting the Internet access.

\section{Acknowledgment}

This research is supported in part by NSF grants CNS$0626609 / 0626614 / 0720465$. We also receive the facility support from MSI and DTC at the University of Minnesota.

\section{References}

[1] A. Carter, "The Status of Vehicle-to-Vehicle Communication as a Means of Improving Crash Prevention Performance," Tech. Rep. 05-0264, 2005, http://www-nrd.nhtsa.dot.gov/pdf/nrd-01/ esv/esv19/05-0264-W.pdf.

[2] V. Naumov and T. R. Gross, "Connectivity-Aware Routing (CAR) in Vehicular Ad Hoc Networks," in INFOCOM. IEEE, May 2007.

[3] J. Zhao and G. Cao, "VADD: Vehicle-Assisted Data Delivery in Vehicular Ad Hoc Networks," IEEE Transactions on Vehicular Technology, vol. 57, no. 3, pp. 1910-1922, May 2008.

[4] A. Skordylis and N. Trigoni, "Delay-bounded Routing in Vehicular Ad-hoc Networks," in MobiHoc. ACM, May 2008.

[5] J. Eriksson, H. Balakrishnan, and S. Madden, "Cabernet: Vehicular Content Delivery Using WiFi," in MOBICOM. ACM, Sep. 2008.

[6] H. Yomogita, "Mobile GPS Accelerates Chip Development," http://techon.nikkeibp.co.jp/article/HONSHI/20070424/ $131605 /$.

[7] L. Pelusi, A. Passarella, and M. Conti, "Opportunistic Networking: Data Forwarding in Disconnected Mobile Ad Hoc Networks," IEEE Communications Magazine, vol. 44, no. 11, pp. 134-141, Nov. 2006.

[8] General Motors Corporation's Vehicle-to-Vehicle (V2V) Communications, http://media.gm.com/servlet/ GatewayServlet?target=http://image.emerald.gm.com/gmnews/ viewmonthlyreleasedetail.do?domain $=74 \&$ docid $=19922$.

[9] Garmin Ltd, "Garmin Traffic," http://www8.garmin.com/traffic/.

[10] N. Wisitpongphan, F. Bai, P. Mudalige, and O. K. Tonguz, "On the Routing Problem in Disconnected Vehicular Ad Hoc Networks," in INFOCOM Mini-symposia. IEEE, May 2007.

[11] J. Jeong, S. Guo, Y. Gu, T. He, and D. Du, "TBD: Trajectory-Based Data Forwarding for Light-Traffic Vehicular Networks," Tech. Rep. 08-040, 2008, http://www.cs.umn.edu/ research/technical_reports.php?page=report\&report_id=08-040.

[12] V. Muchuruza and R. Mussa, "Traffic Operation and Safety Analyses of Minimum Speed Limits on Florida Rural Interstate Highways," in Proceedings of the 2005 Mid-Continent Transportation Research Symposium, Ames, Iowa, USA, Aug. 2005.

[13] J. Liu, F. Sailhan, D. Sacchetti, and V. Issarny, "Group Management for Mobile Ad Hoc Networks: Design, Implementation and Experiment," in MDM. ACM, May 2005.

[14] H. Wu, R. Fujimoto, R. Guensler, and M. Hunter, "MDDV: A Mobility-Centric Data Dissemination Algorithm for Vehicular Networks," in VANET. ACM, Oct. 2004. 